\title{
Sicherheitspolitik im Arktischen Rat? Lieber nicht!
}

\author{
Christoph Humrich
}

\begin{abstract}
Ongoing political, legal, environmental and economic changes open up the Arctic. This has implications for regional security. The article tackles the question whether the preeminent regional institution, the Arctic Council, should include securityrelated topics in its portfolio to deal with the emerging challenges. The article argues that the regional security issues will be taken care of better in other institutions for at least two reasons: dealing with security-related topics might negatively impact the Arctic Council's institutional practice, while at the same time the Arctic Council might not provide the best institutional background needed to cope with the most pertinent issues on the security agenda.
\end{abstract}

Keywords: Arctic, Arctic Council, regional security governance

Schlagworte: Arktis, Arktischer Rat, regionale Sicherheitspolitik

\section{Einleitung}

I n den letzten 25 Jahren hat sich die Nordpolarregion in politischer, rechtlicher, wirtschaftlicher und geophysischer Hinsicht radikal gewandelt. ${ }^{1}$ Politisch hat das Ende des Kalten Krieges zur erfolgreichen Institutionalisierung vielfältiger Zusammenarbeit zwischen Russland und dem Westen geführt - wie etwa im Arktischen Rat (Keskitalo 2004; Young 1998). Das Inkrafttreten des Seerechtsübereinkommens der Vereinten Nationen und dessen Ratifizierung durch sieben der acht Arktisstaaten seit Mitte der 1990er Jahre haben Nutzungsansprüche und Kontrollrechte für den Arktischen Ozean und seine angrenzenden Gewässer verändert (Byers 2014). ${ }^{2}$ Das durch den Klimawandel bedingte Abtauen des arktischen Meereises macht die Arktis für den Schiffsverkehr und die Rohstoffextraktion zugänglicher (Arctic Council 2009). Ökonomische Entwicklungen wie zwischenzeitlich steigende Rohstoffpreise, die absehbare Erschöpfung südlicherer Reserven und ein Anstieg des intra- und interregionalen Warenverkehrs haben das Interesse an der Erschließung der Ressourcen in der Arktis und an ihren Wasserstraßen erhöht (Heininen 2005).

Ressourcenerschließung und leichtere Zugänglichkeit der Arktis sowie die mit ihnen einhergehenden divergierenden Nutzungs- und Regelungsinteressen haben auch sicherheitsrelevante Implikationen. Als eine Expedition im August 2007 eine russische Fahne auf dem Meeresgrund des geografischen Nordpols hinterließ, ging zunächst das Schreckgespenst eines durch Ressourcenreichtum befeuerten Territorialkonflikts durch die Medien. Von einem aufziehenden neuen Kalten Krieg oder möglichen kriegerischen Auseinandersetzungen zwischen den Arktisstaaten war die Rede (vgl. Humrich/Wolf 2011). Das weiterhin kooperative Verhalten der Arktisstaaten hat aber zu einem Abflauen düsterer Vorhersagen geführt. ${ }^{3}$ Stattdessen rückten die Sicherheitsgefährdungen in den Vordergrund, die sich aus der leichteren Zugänglichkeit und stei-

1 Überblicke über die Literatur finden sich in Albert/Wehrmann (2015), Humrich (2011), Young (2011a). Sapper/Weichsel/Humrich (2011) versuchen die verschiedenen Entwicklungen und ihre Implikationen aus multidisziplinärer Perspektive einzufangen.

2 Trotz entsprechender Empfehlungen der jeweiligen Administrationen, zeichnet sich im US-amerikanischen Senat noch nicht die erforderliche Mehrheit für eine Ratifizierung des Seerechtsübereinkommens ab.

3 Dieser Wandel der Wahrnehmung der Arktis lässt sich sogar an einzelnen Beobachtern nachvollziehen, vgl. Borgerson (2008) mit (2013). gendem Verkehr ergeben und sich auf die Sicherung der Grenzen sowie die Durchsetzung souveräner Kontrolle beziehen. So werden Schmuggel, illegale Einwanderung sowie auf den internationalen Terror bezogene Grenzverletzungen, maritime Sicherheit und Infrastrukturschutz als sicherheitspolitische Herausforderungen wahrgenommen. ${ }^{4}$ Die Ukrainekrise und die Verschlechterung des Verhältnisses zwischen Russland und dem Westen haben ab 2014 wieder zwischenstaatliche Konfliktlagen in den Vordergrund der sicherheitspolitischen Wahrnehmung der Arktis gerückt (Rasmussen 2015; Regehr 2014c).

Schon in einem der ersten alarmistischen Aufsätze zur Sicherheitslage in der Arktis war davon die Rede (Borgerson 2008), dass in der Region kein hinreichendes Institutionengefüge bestehe, um aufziehende Konfliktlagen kooperativ zu bearbeiten. Ein institutionelles Vakuum (Le Mière/Mazo 2013: 111), zumindest aber eine institutionelle Leerstelle oder ein Defizit sei mit Hinblick auf die regionale Sicherheitsarchitektur in der Arktis zu verzeichnen (Regehr 2014b; Conley et al. 2012: 37). Zwar hat der Arktische Rat, der eine institutionelle Führungsrolle bei der politischen Gestaltung der Region beansprucht, ein umfassendes Mandat zur Förderung der „Kooperation, Koordination und Interaktion“ zwischen den arktischen Staaten. Belange, die Aspekte militärischer Sicherheit berühren, sind aber per Fußnote im Gründungsdokument des Rates explizit aus dem inhaltlichen Portfolio ausgeschlossen (Arctic Council 1996). Uneinigkeit besteht darüber, ob der Rat diese Selbstbeschränkung aufgeben sollte oder ob arktische Sicherheitspolitik nicht besser institutionell in anderen Arenen aufgehoben wäre. Der vorliegende Beitrag greift diese Debatte auf. Zunächst werden dazu die sicherheitspolitischen Herausforderungen in der Region auf verschiedenen Ebenen verortet. Die beiden darauf folgenden Abschnitte untersuchen, was aus der sicherheitspolitischen Perspektive für den Rat als arktische Sicherheitsinstitution, und was aus der institutionellen Perspektive des Rates gegen die Aufnahme von Sicherheit in dessen Portfolio spricht. Schließlich wird skizziert, wie sich der Rat und andere institutionelle Arenen für Sicherheitspolitik in der Arktis zu einer arktischen Sicherheitsarchitektur ergänzen könnten.

4 Gute Überblicke finden sich in Le Mière/Mazo (2013) und Zellen (2013). 


\section{Regionale Sicherheitsherausforderungen in der Arktis}

Wenn man die Analyse der Sicherheitslage in der Arktis begrifflich an der Theorie regionaler Sicherheitskomplexe orientiert (Buzan/Wæver 2003), wird deutlich, dass die Arktis zumindest im Hinblick auf Machtprojektion und nationale Verteidigung keinen eigenen Sicherheitskomplex darstellt. ${ }^{5}$ Ein regionaler Sicherheitskomplex würde sich dadurch auszeichnen, dass die Sicherheit einzelner arktischer Staaten „nicht getrennt von der aller anderen betrachtet werden kann" (diese und die folgenden zwei Zitate: Buzan/Wæver 2003: 43). Die Sicherheitspolitiken der einzelnen arktischen Staaten sowie die Interventionen der in der Region aktiven globalen Mächte müssten „nur aus regionalen Sicherheitsdynamiken verstanden werden können“. Die Arktis wäre für die Akteure die Arena wo der „überwiegende Teil [sicherheitsrelevanter; $\mathrm{CH}$ ] Handlungen stattfindet". Das ist jedoch nicht der Fall. Vielmehr treffen sich in der Arktis sicherheitsrelevante Handlungen unterschiedlicher Ebenen, die aber entweder über die Arktis hinausreichen oder eher subregionaler Natur sind.

Auf der Ebene der globalen Mächtekonkurrenz bleibt die Arktis ein Theater für die Projektion militärischer Macht. Das betrifft Russland und die USA (Murray 2012). Die Ebene globaler Mächtekonkurrenz überschneidet sich bei der strategischen Bedeutung des Arktischen Ozeans für die nukleare Zweitschlagkapazität mit der enger gefassten Ebene der nationalen Verteidigung Russlands, der USA, aber auch Großbritanniens, ohne darum schon in eine interdependente Sicherheitsbeziehung mit den (anderen) arktischen Staaten zu treten, deren Landesverteidigung sich natürlich auch auf deren Gebiete jenseits des Polarkreises erstreckt. Vielmehr spielt der Arktische Ozean im Rahmen der militärischen Planung der NATO auch eine Rolle im dominanten europäisch-transatlantischen Sicherheitskomplex. Über diesen Sicherheitskomplex und nicht primär über die Arktis ist zum Beispiel Norwegens und Dänemarks Sicherheit mit der Kanadas verkoppelt. Finnland und Schweden nehmen als Nichtmitglieder der NATO eine Sonderstellung ein. Für sie ist die Arktis auf der Ebene subregionaler sicherheitspolitischer Kooperation unter den nordischen Staaten und der "Nordic Balance“ im Rahmen bilateraler Beziehungen zwischen ihnen und Russland relevant (Bailes 2010: 210). Auf allen Ebenen finden sich bereits sicherheitspolitische Kooperationsformen. Allerdings könnte ein regionales Forum in der Arktis im Hinblick auf die nationale Verteidigung und globale Machtkonkurrenz Bedeutung erlangen, wenn die Interaktion dort als vertrauensbildende Maßnahme funktioniert. Schließlich sind alle Ebenen durch die Beziehungen zwischen Russland und westlichen Staaten strukturiert (Berkman/Vylegzhanin 2014).

In den letzten zehn Jahren haben die arktischen Staaten ihre militärischen Kapazitäten in der Arktis modernisiert sowie um- und ausgebaut (Regehr/Buelles 2015; Kraska 2011). Dies ist vielfach als Beleg für eine Verschärfung der Sicherheitslage in der Arktis gedeutet worden. Ein Großteil der militärischen

5 So argumentiert auch Exner-Pirot (2013). Sie sieht aber einen arktischen Sicherheitskomplex im Hinblick auf menschliche Sicherheit und Umweltsicherheit.
Veränderungen lässt sich aber ohne Rückgriff auf sicherheitspolitische Entwicklungen in der Arktis erklären: Vielfach fand ein lang geplanter Austausch von ausmusterungsreifem Gerät statt. Einsparungszwänge in Verteidigungshaushalten erforderten neues Material und Organisationsstrukturen, die für mehrere sicherheitspolitische Funktionen einsetzbar sein sollten (Wezeman 2012; Lasserre et al. 2012). Auch die besonders schlagzeilenträchtigen Veränderungen bei arktischen Militäreinheiten in Russland sind zum großen Teil Modernisierungs- und Umstrukturierungsmaßnahmen (Åtland 2011), die allerdings um einen bisher moderaten Wiederaufbau von Kapazitäten für eine global agierende Macht ergänzt werden (Laruelle 2013). Ein spezifisch regionales Problem entsteht dabei erst als Konsequenz, weil die Militärpräsenz auch die Möglichkeit von ungewollten Zwischenfällen mit sich bringt (Regehr 2014a). Um diese zu vermeiden, bedarf es entsprechender Transparenz und Koordination.

Modernisierung und Umstrukturierung militärischer Kapazitäten antizipieren dennoch auch die sicherheitspolitischen Herausforderungen, die sich aus der leichteren Zugänglichkeit der Arktis und einem dadurch steigenden Verkehr innerhalb, in die und durch die Arktis ergeben werden. Bei den entsprechenden Sicherheits- und Sicherungsmaßnahmen in den Weiten der arktischen Landmassen und des Arktischen Ozeans wirken in allen Arktisstaaten auf verschiedene Art und Weise Militär oder paramilitärische Einheiten der Grenz- und Schutzpolizei, Küstenwachen oder Landesverteidigung mit, weil die zivilen Kräfte damit überfordert wären. Aber auch so können die notwendigen Kapazitäten ohne Synergien aus internationaler Kooperation noch kaum hinreichend aus jeweils nationaler Anstrengung bereitgestellt werden. Auch daraus entsteht Kooperationsbedarf: Um die anderen sicherheitspolitischen Ebenen von der spezifisch regionalen Ebene trennen zu können und somit folgenreiche Fehlwahrnehmungen und Missverständnisse zu vermeiden, muss die Präsenz von Sicherheitskräften, die primär mit grenz-, schifffahrts- und schutzpolizeilichen Aufgaben betraut sind, koordiniert werden. Um kostensparende Synergien zu realisieren, müssen entsprechende Verantwortlichkeiten zur maritimen Sicherheit und Verkehrsüberwachung in der Arktis geklärt werden. Ein Informationsaustausch beziehungsweise die koordinierte Generierung relevanten praktischen Wissens oder technischer Lösungen in diesen Bereichen setzt knappe Mittel effizienter ein. Bedarf für eine sicherheitspolitische Institution besteht also bei Transparenz, Koordination und technischer Kooperation. Interaktion in einer regionalen Institution kann zudem als vertrauensbildende Maßnahme sicherheitspolitisch wirksam werden. Bietet sich dafür der Arktische Rat an?

\section{Sicherheitspolitik und der Arktische Rat}

Trotz des Ausschlusses von militärischer Sicherheit ist der Arktische Rat prinzipiell das einzige Forum aller acht arktischen Staaten, dessen Aktivitäten nicht von vornherein auf bestimmte Funktionen begrenzt sind und das daher im umfassenden sicherheitspolitischen Sinne tätig werden könnte. Die institutionelle Vorgeschichte des Arktischen Rates ist zudem sicherheitspolitisch motiviert. Sie begann mit Gorbatschows berühmter 
Murmansk-Rede, ${ }^{6}$ in welcher er unter anderen weitreichende sicherheitspolitische Vorschläge für die Region unterbreitete (Åtland 2008). Die ideelle Vorgeschichte des Rates ist in Kanada schon älter und antizipierte dort eine arktische Institution, zu deren Aufgaben die Sicherheitskooperation explizit gehören sollte (Axworthy/Dean 2013). Die auf die Murmansk-Rede reagierende finnische Initiative griff Gorbatschows Vorschläge jedoch nur im Hinblick auf Umweltschutz auf. Das schien für eine vertrauensbildende Maßnahme angemessen, weil das auf beiden Seiten noch mit Unsicherheiten belastete neue Verhältnis zwischen Ost und West erst einmal außerhalb des sicherheitspolitischen Kontextes erprobt werden konnte (Scrivener 1989). Die aus der Initiative hervorgehende Kooperation unter der Arctic Environmental Protection Strategy (AEPS) bereitete dann den Boden für die thematisch breitere und etwas stärker institutionalisierte Kooperation im Arktischen Rat (Scrivener 1996). Ausdrücklich erwähnt das Gründungsdokument nur Nachhaltige Entwicklung und Umweltschutz als Kooperationsfelder. Der Rat soll aber grundsätzlich die Kooperation unter den arktischen Staaten fördern. Vor allem die USA bestanden auf dem expliziten Ausschluss von Sicherheit aus der Kooperation, was zu der schon genannten Fußnote zur militärischen Sicherheit im Gründungsdokument führte. Im ersten Jahrzehnt der Zusammenarbeit im Rat war die Empfindlichkeit der USA im Hinblick auf Sicherheit so groß, dass jede Verwendung des Begriffs Sicherheit tabu war: Auch die Nutzung der Konzepte menschlicher Sicherheit oder Umweltsicherheit, die durchaus mit den ausdrücklich erwähnten Kooperationsfeldern vereinbar gewesen wäre, wurde sorgfältig vermieden.

Das Tabu der begrifflichen Verwendung von Sicherheit hat sich in den letzten Jahren langsam abgeschliffen. So hat der Rat auch Maßnahmen beschlossen, die direkt den Bereich militärischer Sicherheit berühren. Die Hoffnung, dass der Arktische Rat den expliziten Ausschluss von Sicherheit aus seinem Portfolio verwirft, stützt sich auf die antizipierte Verlängerung dieser Entwicklung. Außerdem gehen die Befürworter einer sicherheitspolitischen Komponente des Arktischen Rates davon aus, dass die Zusammenarbeit im Rat schon in dem Maße als vertrauensbildende Maßnahme gewirkt hat, dass der Befassung mit Sicherheitsfragen kein grundsätzliches Hindernis in den gegenseitigen Beziehungen mehr entgegensteht (Berkman/Vylegzhanin 2014).

Im Zusammenhang mit der Arbeit des Rates war (menschliche) Sicherheit bereits im Hinblick auf Geschlechterfragen, Gesundheit und Ernährung thematisiert worden (Larsen/Fondahl 2015; Einarsson et al. 2004; vgl. Exner-Pirot 2013). Seit dem Ministertreffen in Tromsø 2009 beinhalteten die Erklärungen des Rates zudem Hinweise auf Frieden und friedliche Konfliktlösung in der Region. Das Tromsøer Treffen setzte als Follow-up eines Berichtes des Rates zur arktischen Schifffahrt auch eine Task-Force ein (Arctic Council 2009), die unter dem Vorsitz der USA und Russlands ein Abkommen zur Verbesserung der maritimem Such- und Rettungskooperation verhandelte. Das sogenannte SAR-Übereinkommen wurde bereits auf dem nächsten Treffen des Rates 2011 in Nuuk unterzeichnet. Auch

6 Der Text der Rede, die am ersten Oktober 1987 anlässlich der Überreichung einer Auszeichnung an die Stadt Murmansk gehalten wurde, ist unter <http://www.arctic.or.kr/files/pdf/m2/m22/1/m22_1_eng.pdf> verfügbar (Zugriff 15.07.2015). wenn gelegentlich Wert darauf gelegt wird, dass das Abkommen nur unter der Schirmherrschaft des Rates (und nicht als Abkommen des Rates) verhandelt wurde, laufen die vom Abkommen ausgehende Kooperation und Aktivitäten im Arktischen Rat zur arktischen Schifffahrt weitgehend zusammen. Das Abkommen zog bereits gemeinsame Übungen nach sich, in die auch militärisches Personal involviert war, und gab den arktischen Stabschefs Anlass zu einem gemeinsamen Treffen zur Such- und Rettungskooperation (Exner-Pirot 2012). ${ }^{7}$ Die USA haben 2015 zum zweiten Mal den Vorsitz im Arktischen Rat übernommen. Ein Schwerpunktthema des Vorsitzes soll maritime Sicherheit sein. Dabei wird im Englischen neben dem engeren und von militärischen Konnotationen weiter entfernten „safety“ offiziell auch der Begriff „security“ verwendet. ${ }^{8}$ Da das Thema zudem über die Aufgaben der Küstenwache und den Verkehr von Marineschiffen den Bereich militärischer Sicherheit berührt, weist das auf einen entspannteren Umgang der USA mit dem Sicherheitsbegriff im institutionellen Kontext des Rates hin. Damit scheint die Tür für militärische Sicherheitskooperation auch im Rahmen des Arktischen Rates geöffnet. Allerdings ist nun aus der Perspektive der bestehenden Kooperation im Arktischen Rat zu fragen, ob eine weitergehende Einbeziehung von Sicherheit auch wünschenswert ist.

\section{Der Arktische Rat und Sicherheitspolitik}

Der Arktische Rat ist zu Recht für sein innovatives institutionelles Konstrukt für die Beteiligung der indigenen Völker gelobt worden (Koivurova/Heinamäki 2006). Zwar ist den Organisationen der arktischen indigenen Völker das Recht auf Mitentscheidung verwehrt, ansonsten genießen sie aber nahezu die gleichen Teilnahmerechte im Rat wie die arktischen Staaten. Schon im Zuge der Debatte um die Forderung nach einem wie auch immer gearteten völkerrechtlich bindenden Rechtsdokument für die Arktis wurde von Gegnern eines solchen Dokumentes eingewandt, dass es vermutlich die politischen Einflussmöglichkeiten der indigenen Völker schwächen würde (Le Mière/Mazo 2013: 151f.; Young 2011b). Dass diese Vermutung durchaus berechtigt ist, zeigt die Erfahrung des SARAbkommens von dessen Verhandlung und Inhalt die indigenen Völker weitgehend ausgeschlossen waren (Wood-Donnelly 2013). Zwar geht es bei der Behandlung von Sicherheitsfragen nicht um den Status der indigenen Völker als Völkerrechtssubjekte, aber Sicherheit ist, wie das Völkerrecht, einer der Bereiche, in dem und über den sich Regierungen Handlungsfreiheiten sichern (Wolf 1999). Sollte sich der Arktische Rat der Sicherheitspolitik annehmen, ist zu befürchten, dass sich die institutionelle Praxis zuungunsten der Einflussmöglichkeiten der indigenen Völker in dem Maße verändern könnte, wie die Staaten Mittel und Wege finden, ihre sicherheitspolitischen Handlungsfreiräume zu wahren. Sollten sie diese Freiräume betreffende Themen aufgrund der Teilnahmerechte indigener Völker gar nicht erst in den Rat bringen, ginge die Befassung mit militärischer Sicherheit wohl kaum über die jetzigen Berührungspunkte hinaus.

7 Erstes Arctic Chiefs of Defense Staff Meeting, 12.-13.04.2012.

8 Siehe: http://www.arctic-council.org/index.php/en/about-us/arcticcouncil/u-s-chairmanship (Zugriff 15.06.2015). 
Teilnehmer und Beobachter attestieren dem Arktischen Rat besonderen Erfolg in den Bereichen technischer und wissenschaftlicher Zusammenarbeit (z.B. Kankaanpää/Young 2012; Stokke/ Hønneland 2007). Die entsprechende Nische, die der Arktische Rat damit im regionalen Institutionengefüge besetzt, ist das Ergebnis eines Lernprozesses, in dem sich die institutionelle Praxis und Struktur des Rates auf projektbasierte und produktorientierte Kooperation in Arbeitsgruppen ausrichtete (Koivurova et al. 2015; Stokke/Hønneland 2007). Auch wenn, wie oben angedeutet, technische Kooperation und Wissensproduktion durchaus ein sinnvoller Bereich regionaler sicherheitspolitischer Kooperation in der Arktis wäre, wäre die Befassung mit militärischer Sicherheit in der bisherigen Praxis und Struktur des Rates durch die Nähe zu politisch sensiblen Fragen vermutlich ein Fremdkörper. Diese Vermutung beruht auf den Erfahrungen des Rates mit dem Thema nachhaltige Entwicklung. Dies war von Anfang an hoch politisiert. Das erschwerte schon die bloße Einigung auf die formellen Rahmenbedingungen sowie das Themenspektrum der Nachhaltigkeitsarbeitsgruppe erheblich und zog die entsprechenden Verhandlungen über Jahre hin (Scrivener 1999; 1996). Zudem brachte die Politisierung eine strikte Kontrolle der Arbeitsgruppe durch die politische Ebene der nationalen Senior Arctic Officials (SAO) mit sich, was den fachlichen Dialog zwischen Experten in der Arbeitsgruppe behinderte. Wenig spricht dafür, dass dies bei einer Verhandlung der Einbeziehung von Sicherheit in den Arktischen Rat und im operativen Geschäft einer entsprechenden Arbeitsgruppe anders wäre. Politische Verhandlungen auf der Ebene der SAO würden dort vermutlich überproportional Kapazitäten binden, den Aktivitätsschwerpunkt von den Arbeitsgruppen weg verlagern und damit die Bearbeitung anderer Themen beeinträchtigen.

Der Fall der Erweiterung der AEPS-Kooperation um nachhaltige Entwicklung legt zudem nahe, dass die Integration von militärischer Sicherheit in den Rat politische Koppelgeschäfte (im Gegensatz zu sachlichen) herausfordern und den Rat zu einem Pfand in der Sicherheitspolitik machen könnte. Solche Koppelgeschäfte hatten Mitte der 1990er Jahre zeitweilig die fachliche Arbeit um nachhaltige Entwicklung in eine politisierte Diskussion verwandelt, jeden sachlichen Fortschritt blockiert und die Verhandlungen im Rat beinahe zum Scheitern gebracht. Der kanadische Boykott eines Arbeitsgruppentreffens des Rates in Russland, die Absage zahlreicher Workshops und gemeinsamer Übungen zwischen Russland und den anderen Arktisstaaten aufgrund der Ukrainekrise geben seit dem letzten Jahr einen Vorgeschmack auf mögliche negative Auswirkungen der Beschäftigung mit Sicherheitspolitik auf die übrige Arbeit des Arktischen Rates (Regehr 2014c). Die anderen westlichen Staaten hatten Mühe, Kanada von der Boykotthaltung abzubringen. Das gelang nur, weil der besondere regionale Wert des Rates mit seinem bisherigen thematischen Fokus gerade in der zirkumpolaren Mitgliedschaft liegt: „G7 statt G8 kann vielleicht funktionieren. Aber Arktische Sieben statt Arktische Acht wären zwecklos" (zitiert in: Regehr 2014b). Eine Integration von Sicherheitspolitik in den Rat würde demnach der Gefahr einer politischen Instrumentalisierung des Rates Vorschub leisten und damit auch die restliche Arbeit als regionales Forum gefährden. Wenn sich der Arktische Rat nicht der besonderen sicherheitspolitischen Herausforderungen der Region annehmen soll, welche Institutionen kommen dann in Frage?

\section{Sicherheitsinstitutionen und regionale Sicherheitsherausforderungen in der Arktis}

Zwar insistierten bei den Verhandlungen zur Schaffung des Arktischen Rates insbesondere die USA auf dem Verbot der Befassung mit militärischer Sicherheit, aber auch die anderen Arktisstaaten stimmten zu, dass Sicherheitspolitik bei NATO und OSZE besser aufgehoben sei (Scrivener 1996). Gegen die NATO als Forum für regionale Sicherheit in der Arktis sprechen jedoch strukturelle und politische Gründe. Als Verteidigungsbündnis ist die NATO strukturell exklusiv und primär auf den Einsatz im militärischen Ernstfall ausgerichtet. Das entspricht nicht dem Anforderungsprofil für die spezifisch regionalen sicherheitspolitischen Herausforderungen (Regehr 2013). Für Koordination, Transparenz und technische Zusammenarbeit ist eine inklusive und operative Institution erforderlich. Selbst der NATO-Russland-Rat spiegelt trotz Konsensprinzip noch die asymmetrischen Beziehungen zwischen den NATO-Mitgliedstaaten auf der einen und zwischen diesen und Russland auf der anderen Seite (Bailes 2010) wider. Die Ausrichtung auf den militärischen Ernstfall ist für die kommenden grenz-, schifffahrts-, und schutzpolizeilichen Aufgaben in der Arktis zu eng und rückt die Allianz bei Krisen zudem sofort in den politischen Fokus. Spannungen mit Russland haben sich darum auch immer unmittelbar auf die Zusammenarbeit im NATO-Rahmen ausgewirkt. Die politischen Gründe gegen die NATO als regionales Forum liegen in der Haltung der einzelnen Arktisstaaten (Haftendorn 2011). Kanada lehnt eine Rolle der Allianz in der Arktis aus Souveränitätsgründen vehement ab. Während die Norweger sich um eine größere Aufmerksamkeit der NATO in Bezug auf die Arktis bemüht haben, ist ihnen auch eine kooperative Beziehung zu Russland strategisch wichtig (Bailes 2010). Russland hat aber mehrfach deutlich gemacht, dass es keine Funktion für die Allianz in der Arktis sieht (Regehr 2013). Sollte die NATO dennoch eine beanspruchen, würde das wohl als weiteres Vordringen des Westens in die Sicherheitssphäre Russlands wahrgenommen und bestehende Vorurteile und Befürchtungen bestätigen.

Die OSZE könnte dagegen eine größere Rolle in der sicherheitspolitischen Institutionenarchitektur der Arktis spielen. Sie ist inklusiv und operativ und befasst sich mit militärischen und polizeilichen Dimensionen von Sicherheit. Gegen eine mögliche Rolle der OSZE in der Arktis ist vorgebracht worden, dass die arktischen Staaten Wert darauf legen, Dritte aus spezifisch regionalen Problemen herauszuhalten (Regehr 2014b). In der Parlamentarischen Versammlung der OSZE wurde aber mit Zustimmung der arktischen Staaten bereits mehrmals die regionale Sicherheit in der Arktis in Resolutionen thematisiert. ${ }^{9}$ Im OSZE-Rahmen fanden auch schon mehrere Aktivitäten zur maritimen Sicherheit und Gefahrenabwehr statt, die einen expliziten Bezug zur Arktis herstellten. Breite Mitgliedschaft und weitgefasste Tätigkeiten und Expertise in der OSZE prädestinieren sie für technische Kooperation im Hinblick auf die kommenden grenz-, schifffahrts-, und schutzpolizeilichen Aufgaben. Schließlich kann, wie Benjamin Schaller (2014) herausgearbeitet hat, der Vertrag über den Offenen Himmel (Open Skies) als Transparenzregime eine wertvolle regionale Funktion erfüllen. Zwar sind seiner Meinung nach auch das Wiener Dokument und der Vertrag über konventionelle Streitkräfte in Europa der Arktis

9 Die Abschlussdokumente der Parlamentarischen Versammlungen 2010 und 2013 enthalten zum Beispiel Entschließungen zur Arktis. 
nützlich, aber Beobachtungsflüge im Luftraum anderer Staaten könnten die Transparenz über die Stationierung und Bewegungen von Landstreitkräften und Marineeinheiten in der Arktis erhöhen. Letzteres trüge vermutlich durch die einhergehende Verbesserung der Kommunikation und des Informationsaustausches langfristig auch zur Vermeidung von ungewollten Zwischenfällen bei, die sich durch die steigenden Präsenz von Marine- und Küstenwachenschiffen mit schifffahrtspolizeilichen Aufgaben in der Arktis ereignen könnten. Entsprechende Ausführungsvorschriften müssten natürlich noch verhandelt werden, aber Schaller zeigt, dass die Vertragsgebiete ohne größeren Aufwand in der Arktis erweitert werden könnten und das OSZE-Regime ausdrücklich zu regionalen Abkommen und Aktivitäten in seinem Rahmen ermuntert. Mit dem OSZE Dokument über vertrauens- und sicherheitsbildende Maßnahmen im Bereich der Seestreitkräfte im Schwarzen Meer existiere außerdem schon eine Vereinbarung, aus der entsprechende Teile als Vorlage dienen könnten (Schaller 2014: 448; Fn. 2).

Die für die Vermeidung von ungewollten Zwischenfällen unmittelbar notwendige kurzfristige Koordination kann demgegenüber besser in regionalen Arenen stattfinden. Die Treffen der arktischen Stabschefs sowie der runde Tisch der arktischen Sicherheitskräfte und das gerade neu gegründete Küstenwachenforum sind die geeigneten Arenen, um dafür Strukturen zu schaffen. ${ }^{10}$ Die notwendige Verteilung von Verantwortung bei der internationalen Dimension kommender grenz-, schifffahrts-, und schutzpolizeilicher Aufgaben sollte in zwischenstaatlichen Arenen verbindlich festgelegt werden, so wie es das schon genannte SAR-Abkommen für seinen Bereich tut. Unter der Schirmherrschaft des Arktischen Rates könnten weitere ähnliche Abkommen folgen. Darüber hinaus hatten sich die Anrainerstaaten des Arktischen Ozeans, USA, Kanada, Dänemark, Norwegen und Russland sich zum Beispiel 2008 im grönländischen Ilulissat getroffen, um sich der gegenseitigen Kooperation und friedlichen Konfliktlösung bei der nur sie betreffenden Aufteilung des Kontinentalschelfs und der Ausübung ihrer souveränen Rechte im Arktischen Ozean mit einer (völkerrechtlich unverbindlichen) Erklärung zu versichern. ${ }^{11}$ Im Juli 2015 wurde zudem eine gemeisame Erklärung der Fünf zur Fischerei unterzeichnet. Weitere Arrangements zu maritimen Fragen scheinen daher denkbar.

Dem Arktischen Rat käme aber noch eine weitere Tätigkeit zu. Die oben genannten Treffen der Sicherheitskräfte können in Zeiten niedriger Spannungen, wie von Heather Exner-Pirot (2012) vorgeschlagen, Aktivitäten der Verteidigungsdiplomatie organisieren. Allerdings zeigen die jüngsten Entwicklungen, dass diese Bereiche wegen ihres militärischen Bezuges nicht immun gegen die Auswirkungen politischer Spannungen sind: Die Treffen der Stabschefs wurden ausgesetzt und der Runde Tisch fand ohne Beteiligung Russlands statt. In der Sicherheitsarchitektur der Arktis fiele dem Arktischen Rat die Aufgabe zu, auch in Krisenzeiten einen offenen Kanal für Kommunikation und vertrauensbildende Kooperation bereitzustellen. Dem

10 Arctic Security Forces Roundtable und Arctic Coast Guard Forum. Conley et al. (2012) arbeiten die Rolle vor allem für das Arctic Coast Guard Forum aus. Regehr (2013) berichtet über die Schritte, die das zweite Arctic Chiefs of Defense Staff in Richtung Sicherheitskoordination erbracht hat.

11 Die Ilulissat-Erklärung ist abgedruckt in Witschel et al. (2010), 293-294. Dort erklären Norwegen, Dänemark, Kanada, die USA und Russland gemeinsam, dass sie das Seerecht als verbindliche Grundlage für die Lösung strittiger Nutzungsfragen ansehen. Einen Überblick mit Interpretation gibt Winkelmann (2008).
Grundgedanken der finnischen Initiative zur regionalen $\mathrm{Zu}$ sammenarbeit unter der AEPS Ende der 1980er Jahre würde es entsprechen, dass der Arktische Rat dies vor allem gewährleistet, indem er auf Sicherheitspolitik in seinem Portfolio verzichtet.

\section{Fazit}

Die Arktis hat auf verschiedenen Ebenen sicherheitspolitische Bedeutung. Dem muss die Sicherheitsarchitektur Rechnung tragen. Die mit der Rolle als Theater für globale Machtprojektion verbundenen sicherheitspolitischen Herausforderungen können nur auf der Ebene bilateraler Kooperation zwischen den USA und Russland sinnvoll bearbeitet werden. Herausforderungen im Zusammenhang mit nationalen Verteidigungsstrategien und im europäisch-transatlantischen Sicherheitskomplex werden am besten innerhalb der NATO und im NATO-Russland-Rat verhandelt. Die durch diese Ebenen bedingte Militärpräsenz schafft aber auch spezifisch regionale Probleme. Diese werden durch Transparenz und Koordination verringert, die in der OSZE und in regionalen Foren der Sicherheitskräfte bereitgestellt werden können. Zusammenarbeit im Rahmen der OSZE kann zudem für kostensparende Synergien durch technische Kooperation sorgen. Verteidigungsdiplomatie in den regionalen Foren der Sicherheitskräfte kann sich in Friedenszeiten auch positiv auf die anderen Ebenen auswirken.

Die Rolle des Arktischen Rates in der regionalen Sicherheitsarchitektur sollte aber vor allem darin bestehen, unter seiner Schirmherrschaft völkerrechtliche Abkommen als Rahmen für die Kooperation zu den regionalen Sicherheitsherausforderungen zu initiieren und einen offenen Kanal für Kommunikation und Kooperation auch in Krisenzeiten zu bieten. Dafür muss er sich von Sicherheitspolitik im engeren Sinne fernhalten. Berührungen mit Sicherheitspolitik wie im Falle des SAR-Abkommens scheinen bisher unproblematisch, weil sie aus dem Kontext der Zusammenarbeit zu Umweltschutz und nachhaltiger Entwicklung erwachsen sind. In Hinblick auf eine Versicherheitlichung anderer Themen im Arktischen Rat durch Einführung konzeptioneller Rahmungen wie menschlicher Sicherheit, Umwelt- und Energiesicherheit sollte aber Vorsicht geboten sein. Die Koppelung mit militärischer Sicherheit wird dadurch möglicherweise forciert. Eine Trennung von nachhaltiger Entwicklung und Umweltschutz auf der einen Seite und von regionalen Sicherheitsproblemen auf der anderen ist besser für die Bearbeitung beider Komplexe: Die drängenden Aufgaben des Umweltschutzes und der Verbesserung der Lebensbedingungen der indigenen Bevölkerung dulden keine Behinderung durch politisierte Sicherheitsdiskussionen. Umgekehrt nützt es der regionalen Sicherheit, wenn der Arktische Rat als eine Arena funktioniert, in der auch in spannungsgeladenen Zeiten Kommunikation und Kooperation möglich bleiben.

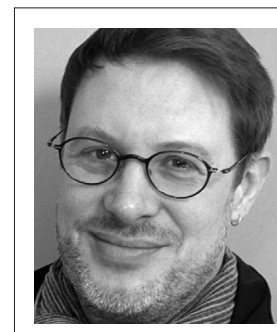

Christoph Humrich ist Assistant-Professor für Internationale Beziehungen und Sicherheitspolitik am Department für Internationale Beziehungen und Internationale Organisation der Universität Groningen sowie Associate Researcher am dortigen Arctic Centre. 


\section{Literatur:}

Albert, Mathias/Wehrmann, Dorothea 2015: Polarpolitik. Ein Bericht zur politikwissenschaftlichen Arktis- und Antarktisliteratur, in: Neue Politische Literatur 60: 1, im Erscheinen.

Arctic Council 1996: Arctic Council Declaration, Ottawa.

Arctic Council 2009: Arctic Marine Shipping Assessment, PAME, Akureyri.

Arctic Governance Project 2010: Arctic Governance in an Era of Transformative Change: Critical Questions, Governance Principles, Ways Forward, ohne Ort.

Åtland, Kristian 2008: Mikhail Gorbachev, the Murmansk Initiative, and the Desecuritization of Interstate Relations in the Arctic, in: Cooperation and Conflict 43: 3, 289-311.

Åtland, Kristian 2011: Im Norden nichts Neues? Die Arktis in Russlands Sicherheitspolitik, in: Osteuropa 61: 2-3, 243-256.

Axworthy, Thomas S./Dean, Ryan 2013: Changing the Arctic Paradigm from Cold War to Cooperation: How Canada's Indigenous Leaders Shaped the Arctic Council, in: The Yearbook of Polar Law Online 5: 1, 7-43.

Bailes, Alyson J. K. 2010: Potential roles of NATO and the EU in High Northern security, in: Yearbook of Polar Law 2: 1, 201-224.

Berkman, Paul Arthur/Vylegzhanin, Alexander N. 2014: Conclusions: Building Common Interests in the Arctic Ocean, 371-404 in: dies. (Hrsg.): Environmental Security in the Arctic Ocean, Dordrecht.

Borgerson, Scott G. 2008: Arctic Meltdown. The Economic and Security Implications of Global Warming, in: Foreign Affairs 87: 2, 63-77.

Borgerson, Scott G. 2013: The Coming Arctic Boom. As the Ice Melts, the Region Heats Up, in: Foreign Affairs 92: 4, 76-89.

Buzan, Barry/Woever, Ole 2003: Regions and powers: the structure of international security, Cambridge.

Byers, Michael 2014: International Law and the Arctic, Cambridge.

Conley, Heather A./Toland, Terry/Kraut, Jamie/Østhagen, Andreas 2012: A New Security Architecture for the Arctic. An American Perspective, Washington/D.C.

Einarsson, Níels/Larsen, Joan Nymand/Nilsson, Annika/Young, Oran R. 2004: Arctic Human Development Report, Stefansson Arctic Institute, Akureyri.

Exner-Pirot, Heather 2012: Defence diplomacy in the Arctic: the search and rescue agreement as a confidence builder, in: Canadian Foreign Policy Journal 18: 2, 195-207.

Exner-Pirot, Heather 2013: What is the Arctic a case of? The Arctic as a regional environmental security complex and the implications for policy, in: The Polar Journal, 1-16.

Haftendorn, Helga 2011: NATO and the Arctic: is the Atlantic Alliance a cold war relic in a peaceful region now faced with nonmilitary challenges?, in: European Security 20: 3, 337-361.

Heininen, Lassi 2005: Impacts of Globalization, and the Circumpolar North in World Politics, in: Polar Geography 29: 2, 91-102.

Humrich, Christoph 2011: Die Zukunft der Arktis. Ein Literaturbericht, in: Osteuropa 61: 2-3, 428-441.

Humrich, Christoph/Wolf, Klaus Dieter 2011: Krieg in der Arktis? Konfliktszenarien auf dem Prüfstand, in: Osteuropa 61: 2-3, 225-242.

Kankaanpää, Paula/Young, Oran R. 2012: The effectiveness of the Arctic Council, in: http://www.polarresearch.net/index.php/ polar/article/view/17176, 2012-10-26.

Keskitalo, Eva Carina Helena 2004: Negotiating the Arctic. The Construction of an International Region, New York.
Koivurova, Timo/Heinamäki, Leena 2006: The participation of indigenous peoples in international norm-making in the Arctic, in: Polar Record 42: 1, 101-109.

Koivurova, Timo/Kankaanpää, Paula/Stępień, Adam 2015: Innovative Environmental Protection: Lessons from the Arctic, in: Journal of Environmental Law, 27: Advance Access.

Kraska, James (Hrsg.) 2011: Arctic Security in an Age of Climate Change, Cambridge.

Larsen, Joan Nymand/Fondahl, Gail 2015: Arctic Human Development Report: Regional Processes and Global Linkages, Nordic Council of Ministers,TemaNord: 567.

Laruelle, Marlene 2013: Russia's Arctic strategies and the future of the Far North, Armonk/NY.

Lasserre, Frederic/Le Roy, Jérôme/Garon, Richard 2012: Is there an arms race in the Arctic?, in: Journal of Military and Strategic Studies 14: $3 \& 4,1-55$.

Le Mière, Christian/Mazo, Jeffrey 2013: Arctic Opening: Insecurity and Opportunity, Abingdon.

Murray, Robert W. 2012: Arctic politics in the emerging multipolar system: challenges and consequences, in: The Polar Journal 2: 1, 7-20.

Pedersen, Torbjørn 2012: Debates over the Role of the Arctic Council, in: Ocean Development and International Law 43: 1, 146-156.

Rasmussen, Anders Fogh 2015: A Place Apart: A Peaceful Arctic No More?, in: Harvard International Review 36: 3, 45-48.

Regehr, Ernie 2013: NATO and the Arctic, The Simons Foundation, Disarming Arctic Security (16.07.2013): http:// www.thesimonsfoundation.ca/projects/disarming-arctic-security (Zugriff 15.06.2015).

Regehr, Ernie 2014a: Close Encounters with the Russian Military: Implications for Arctic Security Cooperation?, The Simons Foundation, Disarming Arctic Security (25.11.2014): http:// www.thesimonsfoundation.ca/projects/disarming-arctic-security (Zugriff 15.06.2015).

Regehr, Ernie 2014b: Putin and the Institutional Deficit in Arctic Security, The Simons Foundation, Disarming Arctic Security (29.05.2014): http://www.thesimonsfoundation.ca/projects/ disarming-arctic-security (Zugriff 15.06.2015).

Regehr, Ernie 2014c: What do the Russian Actions in Ukraine portend for the Arctic?, The Simons Foundation,Disarming Arctic Security (23.04.2014): http://www.thesimonsfoundation. $\mathrm{ca}$ /projects/disarming-arctic-security (Zugriff 15.06.2015).

Regehr, Ernie/Buelles, Annie Claudine 2015: Circumpolar Military Facilities of the Arctic Five, The Simons Foundation, Disarming Arctic Security (aktualisiert März 2015): http://www.thesimonsfoundation. ca/projects/disarming-arctic-security (Zugriff 15.06.2015).

Sapper, Manfred/Weichsel, Volker/Humrich, Christoph (Hrsg.) 2011: Logbuch Arktis. Der Raum, die Interessen und das Recht (= Osteuropa 2-3/2011), Berlin.

Schaller, Benjamin 2014: Confidence- \& Security-Building Measures in the Arctic: The Organization for Security \& Co-operation in Europe as a Role Model for the Area?, 434-451 in: Heininen, Lassi, Exner-Pirot, Heather, Plouffe, Joel (Hrsg.): Arctic Yearbook 2014: Human Capital in the North, Akureyri.

Scrivener, David 1989: Gorbachev's Murmansk Speech: the Soviet Initiative and Western Response, Oslo.

Scrivener, David 1996: Environmental Cooperation in the Arctic: From Strategy to Council, Oslo.

Scrivener, David 1999: Arctic Environmental Cooperation in Transition, in: Polar Record 35: 192, 51-59. 
Stokke, Olav Schram/Hønneland, Geir (Hrsg.) 2007: International Cooperation and Arctic Governance. Regime Effectiveness and Northern Region Building, London.

Wezeman, Siemon T. 2012: Military Capabilities in the Arctic, Stockholm International Peace Research Institute, SIPRI Background Paper.

Winkelmann, Ingo 2008: Feste Spielregeln für die Aufteilung des Arktischen Ozeans, Stiftung Wissenschaft und Politik, SWPAktuell 53, Berlin.

Witschel, Georg/Winkelmann, Ingo/Tiroch, Katrin/Wolfrum, Rüdiger (Hrsg.) 2010: New Chances and New Responsibilities in the Arctic Region, Berlin.

Wolf, Klaus Dieter 1999: The New Raison d'État as a Problem for Democracy in World Society, in: European Journal of International Relations 5: 3, 333-363.
Wood-Donnelly, Corine 2013: The Arctic Search and Rescue Agreement: Text, Framing and Logics, in: The Yearbook of Polar Law Online 5: 1, 299-318.

Young, Oran R. 1998: Creating Regimes. Arctic Accords and International Governance, Ithaca/NY.

Young, Oran R. 2011a: The Future of the Arctic: Cauldron of Conflict or Zone of Peace?, in: International Affairs 87: 1, 185-193.

Young, Oran R. 2011b: If an Arctic Ocean treaty is not the solution, what is the alternative?, in: Polar Record 47: 4, 327-334.

Zellen, Barry Scott (Hrsg.) 2013: The Fast-Changing Arctic. Rethinking Arctic Security for a Warmer World, Calgary.

\section{Afrika im Fokus}

\section{Zwischen Krieg, Sicherheitsgestaltung und Ethik}

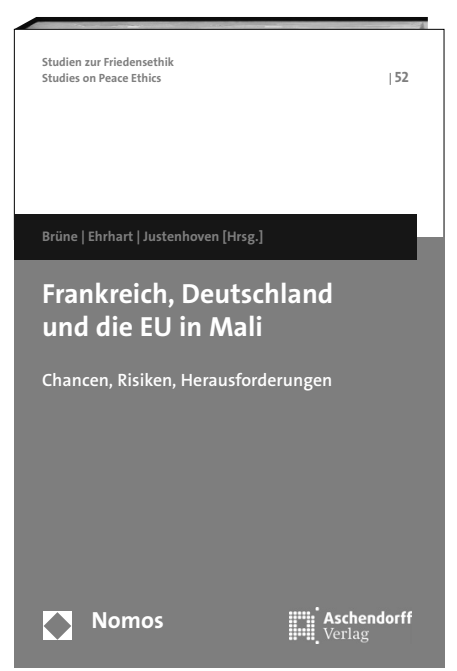

\section{Frankreich, Deutschland und die EU in Mali}

Chancen, Risiken, Herausforderungen

Herausgegeben von Prof. Dr. Stefan Brüne, Dr. Hans-Georg Ehrhart und

Prof. Dr. Heinz-Gerhard Justenhoven

2015, 251 S., geb., 46,- $€$

ISBN 978-3-8487-1895-5

(Studien zur Friedensethik, Bd. 52)

www.nomos-shop.de/23799

Im Oktober 2012 schloss François Hollande den Einsatz französischer Bodentruppen in Mali noch aus, um dann im Januar 2013 einen antiterroristisch begründeten guerre sans merci zu erklären. Für diese Wendung erhielt Frankreich im Blick auf die antizipierte Gefahr internationale Zustimmung und politische Unterstützung für den staatlichen Wiederaufbau Malis.

Dieser mehrsprachige Sammelband untersucht die Gründe für den Meinungsumschwung und fragt nach den politischen Interessen und strategischen Überlegungen für diese Entscheidung. Über den aktuellen Fall hinaus wird untersucht, wie Militäreinsätze zur Beilegung innerafrikanischer Konflikte beitragen können. Die Autoren fragen, in welcher Weise der schwelende Konflikt mit den nordmalischen Bevölkerungsgruppen Ursache der Gewalt war und ob die aktuellen politischen Lösungsvorschläge tragfähig sind. Sie analysieren unter politischen und ethischen Gesichtspunkten die Chancen und Risiken des französischen und europäischen Engagements in Mali.

Bestellen Sie jetzt telefonisch unter 07221/2104-37 